\title{
How Much Time Do Patients with Diabetes Spend
} on Self-Care?

\author{
Monika M. Safford, MD, Louise Russell, PhD, Dong-Churl Suh, PhD, \\ Sheila Roman, MD, and Leonard Pogach, MD
}

Introduction: Little is reported about how much time patients spend on self-management, a cornerstone of diabetes care.

Methods: We studied a cross-section of 1482 diabetic patients enrolled in 3 northeastern United States managed care plans. Patients were surveyed about how much time they spent on foot care, exercise, and food shopping/preparation (7/00 to 9/01). Logistic regressions modeled which types of patients were likely to spend time, and linear regressions modeled characteristics associated with spending more/less time.

Results: Patients (57.9\% $\geq 55$ years; 51.6\% women; 36.2\% African American; 31.1\% on insulin) spent 58 (mean) minutes/day on self-care (interquartile range 19, 84). Many patients skipped individual selfcare elements: $37.9 \%$ reported no foot care, $37.7 \%$ no exercise, and $54.4 \%$ no time on food shopping/ preparation. One fourth of patients with severe foot neuropathy symptoms spent no time on foot care. Never self-testers were less likely than others to engage in foot care (odds ratio (OR) 0.4 (95\% CI 0.3 , 0.6), exercise (OR $0.4(95 \%$ CI $0.3,0.6)$ ), and food shopping/preparation (OR 0.7 (95\% CI 0.5, 1.0)), and were likely to spend 30 minutes less total self-care time than frequent self-testers $(P<.01)$.

Discussion: Most people spent considerable time engaged in self-care, but many skipped individual tasks, offering clinicians opportunities for collaborative decision making. (J Am Board Fam Pract 2005; 18:262-70.)

An important step in collaborative, patient-centered care is acknowledging that chronic disease self-management is time consuming for patients. Diabetes self-care is especially time-demanding, including foot care, exercise, dietary changes, selftesting of glucose, and administration of multiple medications. For patients who suffer from several conditions, each with separate sets of self-care recommendations, the list of self-care tasks can be daunting.

Despite the importance of chronic disease selfcare, little has been reported about how much time

Submitted, revised, 3 March 2005.

From the University of Alabama at Birmingham Preventive Medicine (MMS); Rutgers University (LR, D-CS); Johns Hopkins University (SR); and Veterans Affairs New Jersey Healthcare System (LP).

Funding: MMS and LR were supported by the Centers for Disease Control and Prevention and the National Institute of Diabetes and Digestive Diseases, Cooperative Agreement number U48/CCU216385-01.

Conflict of interest: none declared.

Corresponding author: Monika M. Safford, MD, University of Alabama at Birmingham Preventive Medicine, 1717 11th Avenue South, MT643, Birmingham, AL 35294 (e-mail: msafford@uab.edu).

This work was published in abstract form in the May 2003 issue of the J Gen Intern Med. patients actually spend on their own day-to-day care. Rare reports only mention self-care time demands, ${ }^{1}$ and Singleton noted that "throughout the compliance literature, voices of patients are disturbingly absent." ${ }^{2}$ Thus, little is currently known about how much time patients are spending on chronic disease self-care tasks and which types of patients are likely to spend more or less time.

Knowledge about the time required by selfmanagement is important not only for clinical management but also for economic analysis. For example, cost-effectiveness analyses should consider the impact of the scarce resources of patient time. Despite the recommendation by the Panel on Cost Effectiveness in Health and Medicine that patient and caregiver time be included as a cost of care from the societal perspective, most cost-effectiveness analyses do not include time spent by patients, in part because no data are readily available. ${ }^{3}$

We asked 1482 diabetes patients about the amount of time they spent caring for their feet, exercising, shopping for and preparing special foods, monitoring blood sugar, and taking insulin. Our objective was to quantify the amount of time patients reported spending on each individual ele- 
ment, as well as to address the question of whether there are patient level determinants of time spent on self-care activities.

\section{Methods}

We studied persons with diabetes enrolled in 3 managed care health plans in New Jersey and Pennsylvania. Patients were considered to have diabetes if they had more than one outpatient visit or any inpatient stay with an associated International Classification of Diseases, 9th edition code for diabetes, ${ }^{4}$ or received any diabetes medication. If they met this definition of diabetes and were enrolled in the health plan for at least 12 months, they were invited to participate $(\mathrm{n}=6150)$.

The composition of the final study sample is depicted in Figure 1. Patients were excluded if they did not speak English or Spanish, did not live in the community, were too ill to participate, stated that they did not have diabetes, or reported not receiving most of their health care through the health plan $(\mathrm{n}=2293)$. Of the remaining 3857 people, 2087 were contacted and confirmed eligible, and $85 \%$ of these $(n=1779)$ completed a survey by computer-assisted telephone interview or in writing between July 2000 and September 2001. Census data on neighborhoods of potential participants revealed nominal differences between neighborhoods of responders and nonresponders. As is increasingly common, ${ }^{5}$ despite numerous attempts, many people could not be contacted by phone or mail $(\mathrm{n}=1770)$. If these persons had the same rate of eligibility as those contacted (843 of the 1770) and all declined to participate, the survey response rate would be $61 \%(1779 /(2087+843)) \cdot{ }^{6,7}$ By

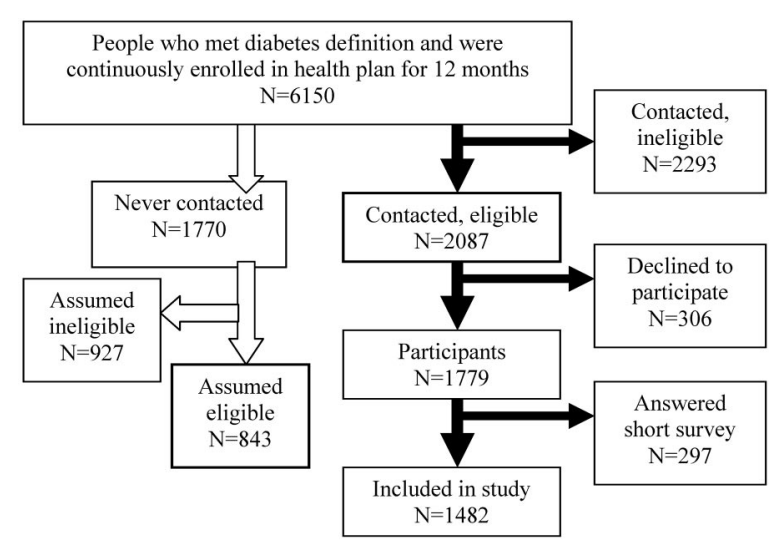

Figure 1. Composition of Final Study Sample. design, some (297) participants were offered a shorter survey that did not include the questions on time; thus 1482 participants are described here.

Because we found no suitable questions in the literature, we created and pilot tested questions about time spent daily on 3 diabetes self-care activities (see Appendix). Because of the length of the survey, we selected activities, based on clinicians' impressions, that were most likely to be important time users and are not done by most people without diabetes. ${ }^{8,9}$ Participants were asked to estimate the extra minutes they spent on a typical day caring for their feet, exercising, and shopping for/preparing food according to diabetes guidelines. To maximize interpretability, the few implausibly extreme outliers were trimmed - at 60 minutes/day for foot care (6 persons), 120 minutes/day for exercise (13 persons), and 180 minutes/day for shopping and cooking foods (5 persons).

Patients were also asked how often they selftested blood glucose and administered insulin. To estimate time spent on these activities, we convened a focus group of 6 certified diabetes educators and asked them to estimate the number of minutes required per event. They estimated that self-testing would take on average 3 minutes per episode, and injecting insulin, including preparation and clean-up time, would take on average 4 minutes per episode. We multiplied their estimates by the number of daily events reported by each patient to estimate total time spent per day on each of these activities.

We summed the times spent on foot care, exercise, food shopping/preparation, self-testing of blood glucose, and taking insulin to estimate total time spent on diabetes self-care. Because we were interested in day-to-day self-care, we did not include time spent attending office visits, obtaining tests, or clarifying insurance issues.

We analyzed bivariate relationships using $\chi^{2}$ or analysis of variance, as appropriate. We used multivariable logistic regressions to model patient characteristics associated with spending any time on each of the 3 activities. For patients who spent any time, we modeled amount of time spent for each activity separately and in total using linear regressions. We wished to examine self-testing frequency as a predictor because self-testing may reflect more severe diabetes or a general motivation to engage in self-care. For modeling of total time, 
we therefore removed time spent self-testing from the response variable.

The analyses examined several types of patient characteristics that might be associated with spending more or less time on self-care. We had 2 objectives: first to confirm that the questions had elicited reasonable responses, and if so, to identify patient level determinants that would allow targeting of intervention. Demographic characteristics included age, sex, income, education, and race/ ethnicity. Measures of general health were the Physical Component Summary (PCS) and Mental Component Summary (MCS) subscores of the Medical Outcomes Study Short Form-12 (SF-12), ${ }^{10}$ and body mass index (BMI). Diabetesspecific health measures included diabetes duration, diabetes medication use, and amputation or neuropathic foot symptoms (as an instance of endorgan damage). We based a foot neuropathy score on the Michigan Neuropathy Screening Instrument. ${ }^{11}$ The score was comprised of 3 questions regarding frequency of (1) numbness or loss of feeling in the feet, (2) tingling or burning sensation in the feet, and (3) sores or wounds on the feet that did not heal. Question responses were: 1, all the time; 2 , most of the time; 3 , some of the time; 4 , little of the time; and 5, none of the time; thus, a lower neuropathy score indicated more frequent symptoms of foot neuropathy (score range 3 to 15 ). Last, we included a measure of health motivation, frequency of self-testing of blood glucose. All analyses were performed on SAS, version 8.2 (SAS Institute Inc., Cary, NC). Our institutional review board approved the study.

\section{Results}

\section{Description of Time Spent in Self-Care}

Although very few $(0.6 \%)$ spent no time at all on self-care, many people did not engage in individual elements: $37.9 \%$ spent no time on foot care, $37.7 \%$ spent no time on exercise, and $54.4 \%$ spent no time on food shopping/preparation. Table 1 depicts the percentage of patients engaging in foot care, exercise, and food shopping/preparation by patient demographic characteristics, in addition to the mean time spent for those who engaged in each activity. Table 2 depicts these same percentages and times

Table 1. Demographic Characteristics of 1482 Persons with Diabetes and Time Spent on Foot Care, Exercise, Food Preparation, and in Total Self-Care

\begin{tabular}{|c|c|c|c|c|c|c|c|}
\hline \multirow[b]{2}{*}{ Characteristic (\%)* } & \multicolumn{2}{|c|}{ Foot Care } & \multicolumn{2}{|r|}{ Exercise } & \multicolumn{2}{|c|}{ Shopping and Cooking } & \multirow{2}{*}{$\begin{array}{c}\text { Total Time Spent }^{\dagger} \\
\text { Mean } \\
\text { min/day } \\
(25 \%, 75 \%)\end{array}$} \\
\hline & $\begin{array}{c}\text { \% Not } \\
\text { Engaging }^{\ddagger}\end{array}$ & $\begin{array}{c}\text { Mean Non-Zero } \\
\text { Time, min/day } \\
(25 \%, 75 \%)\end{array}$ & $\begin{array}{c}\text { \% Not } \\
\text { Engaging }\end{array}$ & $\begin{array}{c}\text { Mean Non-Zero } \\
\text { Time, min/day } \\
(25 \%, 75 \%)\end{array}$ & $\begin{array}{c}\text { \% Not } \\
\text { Engaging }\end{array}$ & $\begin{array}{c}\text { Mean Non-Zero } \\
\text { Time, min/day } \\
(25 \%, 75 \%)\end{array}$ & \\
\hline All Patients & 37.9 & $13(5,15)$ & 37.7 & $32(15,38)$ & 54.4 & $48(30,60)$ & $58(19,84)$ \\
\hline $\begin{array}{l}\text { Age, years } \\
<45(15.1 \%) \\
45 \text { to } 54(27.0 \%) \\
55 \text { to } 64(32.2 \%) \\
>65(25.7 \%)\end{array}$ & $\begin{array}{l}35.9 \\
39.6 \\
37.9 \\
37.2\end{array}$ & $\begin{array}{l}10(5,15)^{\S} \\
12(5,15)^{\S} \\
13(5,15)^{\S} \\
17(5,30)^{\S}\end{array}$ & $\begin{array}{l}37.7 \\
34.8 \\
37.5 \\
41.0\end{array}$ & $\begin{array}{l}33(15,38)^{\S} \\
33(15,40)^{\S} \\
34(20,45)^{\S} \\
28(15,30)^{\S}\end{array}$ & $\begin{array}{l}51.2 \\
53.0 \\
57.5 \\
54.1\end{array}$ & $\begin{array}{l}44(20,60)^{\S} \\
44(20,60)^{\S} \\
47(20,60)^{\S} \\
55(30,60)^{\S}\end{array}$ & $\begin{array}{l}56(21,77) \\
60(21,87) \\
58(19,80) \\
58(15,89)\end{array}$ \\
\hline $\begin{array}{l}\text { Sex } \\
\quad \text { Female }(51.6 \%) \\
\text { Male }(48.3 \%)\end{array}$ & $\begin{array}{l}32.3^{\S} \\
43.8^{\S}\end{array}$ & $\begin{array}{l}14(5,20) \\
13(5,15)\end{array}$ & $\begin{array}{l}40.7^{\S} \\
34.4^{\S}\end{array}$ & $\begin{array}{l}29(15,30)^{\S} \\
35(15,45)^{\S}\end{array}$ & $\begin{array}{l}46.0^{\S} \\
63.6^{\S}\end{array}$ & $\begin{array}{l}51(30,60)^{\S} \\
42(15,60)^{\S}\end{array}$ & $\begin{array}{l}63(22,90)^{\S} \\
53(15,75)^{\S}\end{array}$ \\
\hline $\begin{array}{l}\text { Education } \\
<\text { High school }(52.6 \%) \\
\text { Some college }(25.5 \%) \\
>4 \text { years college } \\
\quad(21.4 \%)\end{array}$ & $\begin{array}{l}35.8^{\S} \\
35.5^{\S} \\
45.3^{\S}\end{array}$ & $\begin{array}{r}15(5,20)^{\S} \\
13(5,15)^{\S} \\
9(5,10)^{\S}\end{array}$ & $\begin{array}{l}41.5^{\S} \\
34.9^{\S} \\
31.7^{\S}\end{array}$ & $\begin{array}{l}31(15,30) \\
33(18,45) \\
33(15,45)\end{array}$ & $\begin{array}{l}51.8^{\S} \\
53.9^{\S} \\
61.6^{\S}\end{array}$ & $\begin{array}{l}52(30,60)^{\S} \\
46(20,60)^{\S} \\
38(15,60)^{\S}\end{array}$ & $\begin{array}{l}60(19,89)^{\S} \\
59(20,82)^{\S} \\
52(18,73)^{\S}\end{array}$ \\
\hline $\begin{array}{l}\text { Ethnicity } \\
\text { African American } \\
(36.2 \%)\end{array}$ & $29.9^{\S}$ & $17(5,20)^{\S}$ & 36.6 & $32(15,30)$ & $49.4^{\S}$ & $52(30,60)^{\S}$ & $65(22,95)^{\S}$ \\
\hline $\begin{array}{l}\text { White (47.0\%) } \\
\text { Others (13.4\%) }\end{array}$ & $\begin{array}{l}43.1^{\S} \\
40.9^{\S}\end{array}$ & $\begin{array}{l}10(5,10)^{\S} \\
14(5,15)^{\S}\end{array}$ & $\begin{array}{l}37.4 \\
42.1\end{array}$ & $\begin{array}{l}33(15,45) \\
31(15,30)\end{array}$ & $\begin{array}{l}60.9^{\S} \\
44.8^{\S}\end{array}$ & $\begin{array}{l}39(15,60)^{\S} \\
55(30,60)^{\S}\end{array}$ & $\begin{array}{l}51(16,74)^{\S} \\
65(24,93)^{\S}\end{array}$ \\
\hline
\end{tabular}

* Percentage of the total study sample $(\mathrm{n}=1482)$ with this characteristic. For example, $36.2 \%$ of the sample was African American.

${ }^{\dagger}$ Total time spent is for the 3 listed elements plus self-testing and insulin injection (see text).

₹ Percentage of the individuals with each characteristic that did not engage in this activity. For example, 29.9\% of African Americans did not engage in foot care.

${ }^{\S} P<.05$. 
Table 2. Clinical Characteristics of 1482 Persons with Diabetes and Time Spent in Foot Care, Exercise, Shopping and Cooking, and in Total Self-Care

\begin{tabular}{|c|c|c|c|c|c|c|c|}
\hline \multirow[b]{2}{*}{ Characteristic $(\%)^{*}$} & \multicolumn{2}{|c|}{ Foot Care } & \multicolumn{2}{|c|}{ Exercise } & \multicolumn{2}{|c|}{ Shopping and Cooking } & \multirow{2}{*}{$\begin{array}{c}\text { Total Time Spent } \\
\text { Mean } \\
\text { min/day } \\
(25 \%, 75 \%)\end{array}$} \\
\hline & $\begin{array}{c}\% \text { Not } \\
\text { Engaging }^{\ddagger}\end{array}$ & $\begin{array}{l}\text { Mean Non-Zero } \\
\text { Time, min/day } \\
(25 \%, 75 \%)\end{array}$ & $\begin{array}{l}\text { \% Not } \\
\text { Engaging }\end{array}$ & $\begin{array}{l}\text { Mean Non-Zero } \\
\text { Time, min/day } \\
\text { (25\%, } 75 \%)\end{array}$ & $\begin{array}{l}\% \text { Not } \\
\text { Engaging }\end{array}$ & $\begin{array}{l}\text { Mean Non-Zero } \\
\text { Time, min/day } \\
(25 \%, 75 \%)\end{array}$ & \\
\hline All patients & 37.9 & $13(5,15)$ & 37.7 & $32(15,38)$ & 54.4 & $48(30,60)$ & $58(19,84)$ \\
\hline $\begin{array}{l}\text { BMI, } \mathrm{kg} / \mathrm{m}^{2} \\
\quad<30(46.3 \%) \\
30 \text { to } 34(27.6 \%) \\
35 \text { to } 40(14.5 \%) \\
>40(11.5 \%)\end{array}$ & $\begin{array}{l}38.3 \\
39.5 \\
36.9 \\
33.5\end{array}$ & $\begin{array}{l}13(5,15) \\
14(5,20) \\
14(5,20) \\
12(5,15)\end{array}$ & $\begin{array}{l}32.6^{\S} \\
35.2^{\S} \\
46.8^{\S} \\
52.4^{\S}\end{array}$ & $\begin{array}{l}34(15,45) \\
32(15,38) \\
31(15,45) \\
26(15,30)\end{array}$ & $\begin{array}{l}53.9 \\
54.9 \\
49.8 \\
61.0\end{array}$ & $\begin{array}{l}46(25,60) \\
47(30,60) \\
49(25,60) \\
52(30,60)\end{array}$ & $\begin{array}{l}59(20,85) \\
59(22,89) \\
57(15,85) \\
51(15,74)\end{array}$ \\
\hline $\begin{array}{l}\text { Neuropathy symptom } \\
\text { Most }(13.5 \%) \\
\text { Moderate }(14.2 \%) \\
\text { Mild }(21.9 \%) \\
\text { None }(44.5 \%)\end{array}$ & $\begin{array}{l}24.1^{\S} \\
30.1^{\S} \\
35.9^{\S} \\
45.5^{\S}\end{array}$ & $\begin{array}{l}16(5,20)^{\S} \\
17(5,20)^{\S} \\
13(5,15)^{\S} \\
11(5,15)^{\S}\end{array}$ & $\begin{array}{l}38.1^{\S} \\
46.2^{\S} \\
33.6^{\S} \\
37.2^{\S}\end{array}$ & $\begin{array}{l}33(15,45) \\
33(15,30) \\
29(15,30) \\
33(15,45)\end{array}$ & $\begin{array}{l}46.3^{\S} \\
48.5^{\S} \\
52.8^{\S} \\
59.7^{\S}\end{array}$ & $\begin{array}{l}46(20,60) \\
53(30,60) \\
45(30,60) \\
44(20,60)\end{array}$ & $\begin{array}{l}66(30,89)^{\S} \\
63(20,94)^{\S} \\
58(19,85)^{\S} \\
53(15,77)^{\S}\end{array}$ \\
\hline $\begin{array}{c}\text { Diabetes treatment } \\
\text { Diet only }(5.5 \%) \\
\text { Oral only }(63.4 \%) \\
\text { Insulin }(31.1 \%)\end{array}$ & $\begin{array}{l}42.0^{\S} \\
41.9^{\S} \\
29.0^{\S}\end{array}$ & $\begin{array}{l}14(5,15) \\
13(5,15) \\
14(5,15)\end{array}$ & $\begin{array}{l}34.2 \\
38.8 \\
35.9\end{array}$ & $\begin{array}{l}29(18,30) \\
33(15,45) \\
32(15,30)\end{array}$ & $\begin{array}{l}40.3^{\S} \\
57.4^{\S} \\
50.6^{\S}\end{array}$ & $\begin{array}{l}46(20,60) \\
48(30,60) \\
46(30,60)\end{array}$ & $\begin{array}{l}55(16,80)^{\S} \\
55(15,79)^{\S} \\
66(24,90)^{\S}\end{array}$ \\
\hline $\begin{array}{l}\text { Self-testing } \\
\text { Never }(20.8 \%) \\
<1 / \text { day }(27.0 \%) \\
\text { 1/day }(12.3 \%) \\
>2 / \text { day }(39.8 \%) \\
\text { Others }(13.4 \%)\end{array}$ & $\begin{array}{l}50.2^{\S} \\
42.1^{\S} \\
35.2^{\S} \\
29.4^{\S} \\
40.9^{\S}\end{array}$ & $\begin{array}{l}12(5,15) \\
13(5,15) \\
14(5,15) \\
14(5,15) \\
14(5,15)^{\S}\end{array}$ & $\begin{array}{l}47.3^{\S} \\
43.0^{\S} \\
33.5^{\S} \\
30.5^{\S} \\
42.1\end{array}$ & $\begin{array}{l}29(15,30) \\
31(15,30) \\
35(20,60) \\
33(15,40) \\
31(15,30)\end{array}$ & $\begin{array}{l}60.4^{\S} \\
55.8^{\S} \\
53.9^{\S} \\
50.5^{\S} \\
44.8^{\S}\end{array}$ & $\begin{array}{l}50(20,60) \\
45(20,60) \\
48(20,60) \\
48(30,60) \\
55(30,60)^{\S}\end{array}$ & $\begin{array}{l}42(4,64)^{\S} \\
47(9,68)^{\S} \\
66(23,87)^{\S} \\
71(35,95)^{\S} \\
65(24,93)^{\S}\end{array}$ \\
\hline
\end{tabular}

* Percentage of the total study sample $(\mathrm{n}=1482)$ with this characteristic. For example, $31.1 \%$ of the sample used insulin.

${ }^{\dagger}$ Total time spent is for the three listed elements plus self-testing and insulin injection (see text).

₹ Percentage of individuals with each characteristic that did not engage in this activity. For example, $29.0 \%$ of insulin-users did not engage in foot care.

"Based on foot neuropathy symptom score (see text). Most symptoms $=3$ to 10 ; moderate $=11$ to $12 ;$ mild $=13$ to $14 ;$ none $=15$. $\$ P<.05$.

by patient clinical characteristics. More women than men engaged in all 3 activities. More people with symptomatic foot neuropathy engaged in foot care, and fewer morbidly obese engaged in exercise and food shopping/preparation. Fully $11.4 \%$ of amputees and $24.1 \%$ of those with the most foot neuropathy symptoms did not engage in foot care. Of the 4 amputees not engaging in foot care, 2 had toe amputations, 1 had a single foot/leg amputation and 1 did not specify.

The amount of time spent on each of these 3 activities varied across a broad range for each task (Figure 2), and this variation persisted among all patient subgroups (Tables 1 and 2). For patients engaging in each activity, the means and interquartile ranges are presented in the tables; median time spent on foot care was 10 minutes, on exercise 30 minutes, and on food shopping/preparation 30 minutes. Tables 1 and 2 also present total time spent daily (ie, the sum of time spent on foot care, exercise, food shopping/preparation, insulin injection, and self-testing). These 1482 patients spent a median of 48 minutes daily on total self-care, with only 9 patients reporting no time engaged in any of these self-care activities.

\section{Predictors of Time Spent on Individual Self-Care Activities}

Table 3 presents the adjusted odds ratios from multivariable logistic regressions for engaging/not engaging in each activity. On average, otherwise similar never or rare self-testers were half as likely as frequent self-testers to engage in foot care or exercise. Amputees were 4.7 times as likely to engage in foot care as non-amputees, those without neuropathy symptoms were half as likely as those with the most neuropathy symptoms, and men were less likely than women. The heaviest were half as likely to exercise as those who were more mildly obese. Men were less likely than women to engage in food shopping/preparation. Whites were half as likely to engage in foot care as African Americans, and also were less likely to engage in food shopping/preparation. 


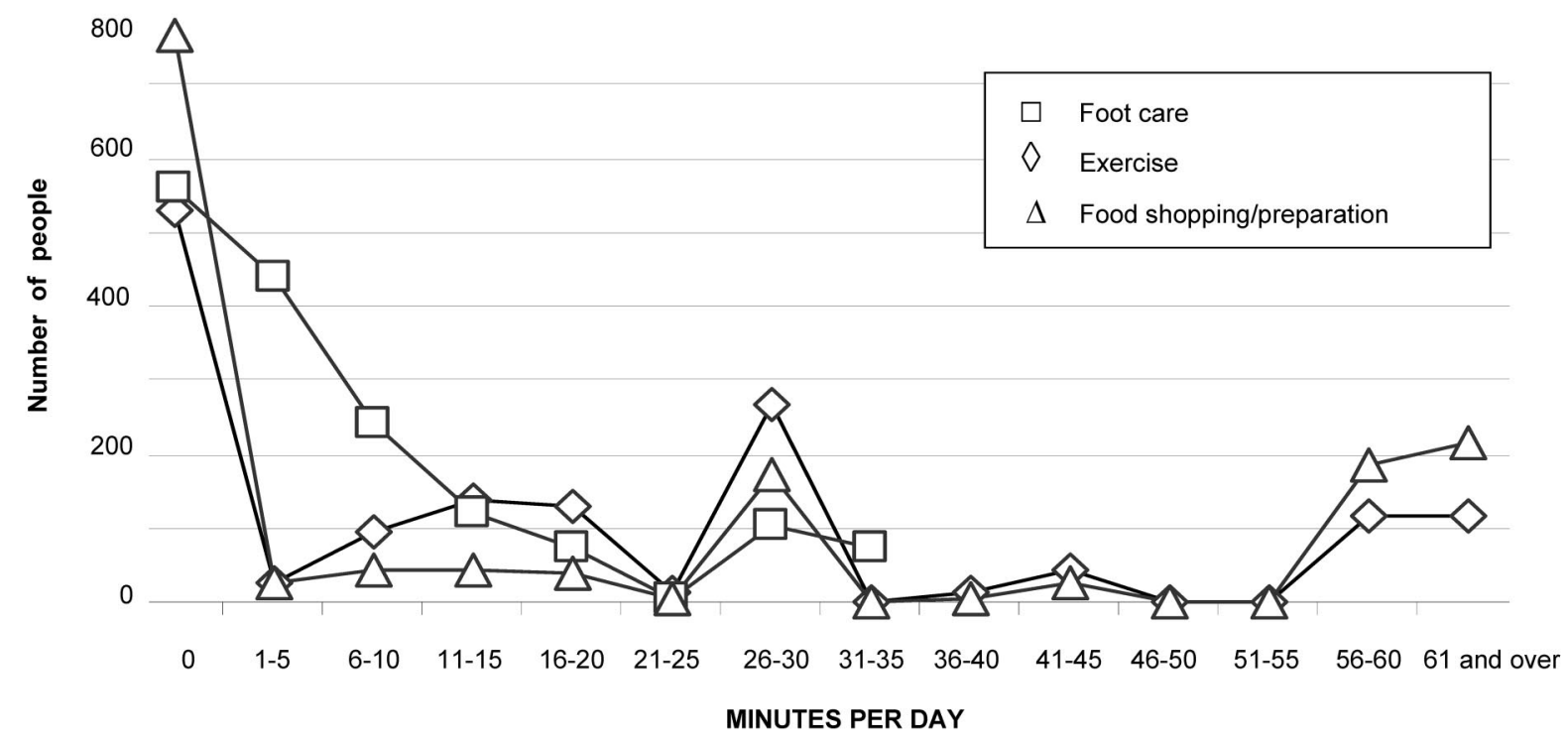

Figure 2. Number of People Reporting Various Times Spent on Foot Care, Exercise, and Food Shopping/Preparation, Minutes per Day.

For those who did engage in each activity, linear regressions for the time spent on each task are presented in Table 4. On average, otherwise similar people who never self-tested spent 3.5 minutes less on foot care and rare self-testers spent 9.1 minutes less on food shopping/preparation than those who tested more than twice daily. The morbidly obese who exercised spent 8 minutes less than patients with BMI 30 to $34 \mathrm{~kg} / \mathrm{m}^{2}$, and women who exer- cised spent 5.1 minutes less than men. Whites spent 4.2 minutes less on foot care, 4.3 minutes less exercising, and 7.7 minutes less on food shopping/ preparation than otherwise similar African Americans.

\section{Predictors of Total Time Spent}

In multivariable analysis, self-testing was associated with the largest difference in total time spent, with

Table 3. Adjusted Odds Ratios* and 95\% CI for Spending Any Time Daily on Foot Care, Exercise, and Food Shopping/Preparation

\begin{tabular}{|c|c|c|c|}
\hline Contrast & Foot Care & Exercise & Food Shopping/Preparation \\
\hline Sex: male vs female & $0.7(0.5,0.9)^{\dagger}$ & $1.1(0.9,1.5)$ & $0.5(0.4,0.7)$ \\
\hline $\begin{array}{l}\text { Body mass index }\left(\mathrm{kg} / \mathrm{m}^{2}\right) \\
>40 \text { vs } 30 \text { to } 34 \\
35 \text { to } 40 \text { vs } 30 \text { to } 34\end{array}$ & $\begin{array}{l}1.1(0.7,1.6) \\
1.1(0.7,1.6)\end{array}$ & $\begin{array}{l}0.5(0.4,0.9) \\
0.6(0.4,0.9)\end{array}$ & $\begin{array}{l}0.7(0.4,1.0) \\
1.4(1.0,2.1)\end{array}$ \\
\hline Amputees vs non-amputees & $4.7(1.3,16.4)$ & $1.3(0.6,3.0)$ & $2.2(1.0,5.0)$ \\
\hline Foot neuropathy: no symptoms vs most & $0.5(0.4,0.8)$ & $1.1(0.7,1.6)$ & $0.7(0.5,1.1)$ \\
\hline Physical functioning: best vs worst quartile & $0.7(0.4,1.0)$ & $1.3(0.9,2.0)$ & $0.8(0.5,1.3)$ \\
\hline Mental functioning: best vs worst quartile & $1.0(0.6,1.4)$ & $1.2(0.8,1.7)$ & $0.7(0.5,4.0)$ \\
\hline $\begin{array}{l}\text { Self-testing } \\
\text { Never vs } \geq 2 \text { times daily } \\
\text { Rarely ( }<\text { daily) vs } \geq 2 \text { times daily }\end{array}$ & $\begin{array}{l}0.4(0.3,0.6) \\
0.5(0.4,0.7)\end{array}$ & $\begin{array}{l}0.4(0.3,0.6) \\
0.5(0.4,0.7)\end{array}$ & $\begin{array}{l}\mathbf{0 . 7}(\mathbf{0 . 5}, \mathbf{1 . 0}) \\
0.9(0.6,1.2)\end{array}$ \\
\hline $\begin{array}{l}\text { Race/ethnicity } \\
\text { Whites vs African Americans } \\
\text { Other races vs African Americans }\end{array}$ & $\begin{array}{l}0.5(0.4,0.7) \\
0.6(0.4,0.9)\end{array}$ & $\begin{array}{l}0.7(0.5,1.0) \\
\mathbf{0 . 6}(\mathbf{0 . 4}, \mathbf{0 . 9})\end{array}$ & $\begin{array}{l}0.7(0.5,0.9) \\
1.1(0.8,1.7)\end{array}$ \\
\hline
\end{tabular}

* Each column presents adjusted odds ratios from a separate logistic regression model that also included age, sex, body mass index, diabetes duration, amputation status, neuropathy symptom score, Physical Component Summary-12 quartile, Mental Component Summary-12 quartile, diabetes treatment, self-testing frequency, education, income, and race/ethnicity. Only predictors with significant findings on at least 1 element are presented.

${ }^{\dagger}$ Adjusted odds ratios in bold had $P<.05$. 
Table 4. Multiple Linear Regression Coefficients $(P) *$ for Minutes Spent on Daily Foot Care, Exercise, Food Shopping/Preparation and Total Time Spent by Patients Spending Any Time on Each Activity

\begin{tabular}{|c|c|c|c|c|}
\hline Contrast & Foot Care $(P)^{\dagger}$ & Exercise $(P)$ & Food Shopping/Preparation $(P)$ & Total Time Spent $(P)$ \\
\hline \multicolumn{5}{|l|}{ Age } \\
\hline$<45$ vs $\geq 65$ years & $-3.7(.02)$ & $3.9(.26)$ & $-9.5(.08)$ & $-6.9(.22)$ \\
\hline 45 to $5 \overline{4}$ vs $\geq 65$ years & $-1.3(.33)$ & $5.0(.10)$ & $-7.4(.14)$ & $0.6(.90)$ \\
\hline Sex: male vs female & $0(.98)$ & $5.5(<.01)$ & $-1.4(.66)$ & $-5.6(.07)$ \\
\hline Body mass index $>40$ vs 30 to $34 \mathrm{~kg} / \mathrm{m}^{2}$ & $-2.6(.06)$ & $-8.0(.02)$ & $9.3(.09)$ & $-11.6(.02)$ \\
\hline Amputees vs non-amputees & $11.4(<.01)$ & $1.1(.84)$ & $-11.7(.14)$ & $16.2(.09)$ \\
\hline Foot neuropathy: no symptoms vs most & $-2.3(.07)$ & $-2.6(.34)$ & $3.2(.50)$ & $-4.5(.35)$ \\
\hline \multicolumn{5}{|l|}{ Physical functioning } \\
\hline Best vs worst quartile & $-3.8(<.01)$ & $7.1(.02)$ & $1.1(.83)$ & $0.50(.92)$ \\
\hline Second best vs worst quartile & $-4.1(<.01)$ & $0.8(.76)$ & $3.0(.50)$ & $0.1(.99)$ \\
\hline Third best vs worst quartile & $-2.8(.02)$ & $-3.2(.24)$ & $4.0(.35)$ & $0.8(.85)$ \\
\hline \multicolumn{5}{|l|}{ Self-testing } \\
\hline Never vs $\geq 2$ times daily & $-3.5(<.01)$ & $-3.6(.19)$ & $-5.7(.21)$ & $-29.9(<.01)$ \\
\hline Rarely ( $<$ daily) vs $\geq 2$ times daily & $-2.6(.02)$ & $-3.8(.10)$ & $-9.1(.01)$ & $-26.8(<.01)$ \\
\hline \multicolumn{5}{|l|}{ Education } \\
\hline$>4$ years college vs $\leq$ high school & $-2.8(.02)$ & $-2.7(.27)$ & $-7.0(.11)$ & $-6.8(.10)$ \\
\hline \multicolumn{5}{|l|}{ Income } \\
\hline$>\$ 75,000$ vs $\$ 40$ to 75,000 & $-3.5(<.01)$ & $-3.0(.21)$ & $-3.6(.43)$ & $-5.9(.15)$ \\
\hline \multicolumn{5}{|l|}{ Race/ethnicity } \\
\hline White vs African Americans & $-4.2(<.01)$ & $-4.3(.05)$ & $-7.7(.03)$ & $-15.1(<.01)$ \\
\hline Other races vs African Americans & $-0.05(.72)$ & $-5.2(.08)$ & $9.5(.04)$ & $-3.1(.52)$ \\
\hline
\end{tabular}

* Coefficients in bold had $P<.05$.

${ }^{\dagger}$ Each column presents results of separate linear regression models including age, sex, body mass index, diabetes duration, amputation status, neuropathy symptom score, Physical Component Summary-12 quartile, Mental Component Summary-12 quartile, diabetes treatment, self-testing frequency, education, income and race/ethnicity. Only predictors with significant findings on at least one element are presented.

otherwise similar never-testers spending $29.9 \mathrm{~min}$ utes less than those self-testing at least twice daily. Whites spent 15.1 minutes less than otherwise similar African Americans. The morbidly obese spent 13.2 minutes less than otherwise similar people with BMI 30 to $34 \mathrm{~kg} / \mathrm{m}^{2}$. Men tended to spend a modest amount less than otherwise similar women (5.6 minutes, $P=.073$ ), amputees tended to spend 16.2 minutes more than non-amputees $(P=.089)$, and those with at least 4 years of college tended to spend 6.8 minutes less than those with high school or less $(P=.099)$. Other patient characteristics did not independently predict total time spent in selfcare (age, diabetes duration, neuropathic symptoms, physical and mental functioning, diabetes treatment, and income).

\section{Discussion}

In this managed care population, people with diabetes reported spending considerable time on diabetes self-care; $75 \%$ of patients spent at least 19 minutes daily, the mean was almost an hour, and the median was 48 minutes each day. The distribution of time among these activities varied consid- erably, with more than one third to one half not engaging in important individual elements of diabetes self-care. Perhaps the most striking finding of this study was the strong association between selftesting frequency and total self-care time. Frequent self-testers were not only more likely to engage in other self-care activities, they were also likely to spend more time on them than those who did not self-test.

Self-testing of blood sugar is recommended for diabetic people treated with insulin, but the role of self-testing is less clear for those who are dietcontrolled or only on oral medications. ${ }^{12}$ To reflect differential self-testing frequency by stage of diabetes, our analyses controlled for use of insulin and oral agents and also to some extent for severity of illness by including foot neuropathy symptoms as well as health status. We demonstrated that for any given level of diabetes treatment or health status, on average, frequent self-testers were more than twice as likely to engage in foot care or exercise and also more likely to spend extra time on food shopping and preparation. In addition, frequent selftesters spent 30 more minutes daily on total self- 
care than never self-testers, a clinically important amount of time. Although a causal relationship between self-testing and engaging in other self-care activities is not warranted from this observational study, the strong association suggests that self-testing may reflect activation to engage in other aspects of self-care.

Another important finding of this study was that people with specific risks were often omitting important elements of self-care. For example, although people with the most severe neuropathic symptoms were twice as likely to spend time caring for their feet as those without symptoms, 1 in 4 patients with severe foot neuropathy symptoms reported spending no time caring for their feet. Foot neuropathy is a dominant risk factor for amputation, and self-care of the feet is one of the most important preventive measures available. ${ }^{12,13}$ Furthermore, although exercise is recommended for all people, it is especially important for weight loss, yet the heaviest people were half as likely as the lightest to engage in exercise, and if they did, they were likely to spend substantially less time exercising. Diabetes care teams could explicitly determine time spent on elements of self-care to uncover such lapses.

In addition, some patients may benefit from redistributing their self-care time. All 171 severe foot neuropathy patients spent at least 15 minutes on total self-care, with a mean of 66 minutes. The 43 spending no time on foot care could reorganize self-care time to include foot care, which often takes only a few minutes. Similarly, $25 \%$ of women spent more than 20 minutes daily on foot care, but women also spent less time exercising than men. In discussions of time management with their diabetic patients, clinicians may uncover excess time devoted to one activity that could be better spent on another, achieving a mix of self-care activities more likely to maximize impact on quality of life and consistent with Donabedian's recommendation that the care team "work diligently with the patient to arrive at a solution that is ultimately acceptable to the patient but is not entirely undirected." 14 Importantly, providers should consider the selfcare burden imposed on persons with diabetes in making self-care recommendations.

This study confirmed that some patients may take more time to accomplish certain tasks, impacting total self-care time requirements. For example, older and more infirm patients spent more time caring for their feet $(P<.05)$ and older patients tended to spend more time in food shopping/ preparation $(P<.10)$. These extra time requirements should be considered in designing treatment plans, especially in the context of the variable benefit older patients can derive from some diabetes interventions, such as tight glycemic control. ${ }^{15}$

In this population, African Americans were more likely than Whites or other races to report engaging in aspects of self-care, and when they did, they spent more time than Whites. African American VA users with diabetes at high risk for amputations were reported to engage in more self-care activities than Whites even after controlling for multiple confounders. ${ }^{16}$ Similarly, another study found high rates of adherence with health recommendations in community-dwelling older African American women. ${ }^{17}$ Given reported health outcome disparities, these intriguing findings warrant further study.

Social and cultural influences undoubtedly accounted for some findings. Whites in this sample were likely to spend the least amount of time on food shopping/preparation, and non-White/nonAfrican Americans were likely to spend the most. Furthermore, although we did not have marital status available, men were half as likely to spend time on food shopping/preparation as women, possibly because wives handled this task for them. When they did spend any time, men spent about the same time as women.

Our study has several limitations. Importantly, self-reports do not necessarily reflect actual time spent, and the request to report "extra" time spent because of diabetes may have caused underestimates of total time. The distributions on reported times had spikes at 30 and 60 minutes, reflecting a common tendency to round off estimates. Nevertheless, the reported times were for the great majority in a plausible range. Indeed, surveying patients about time spent on various activities is a methodology that has been used in social research for decades. ${ }^{18,19}$ The Bureau of Labor Statistics recently launched the American Time Use Survey, ${ }^{18}$ like ours based on telephone interviews. Although less accurate than time use diaries, stylized questions like those used here have been reported to yield similar general estimates. ${ }^{19}$ Alternative approaches to assessing exercise have been used elsewhere, but the distributions we found suggested that our approach worked reasonably well. ${ }^{20} \mathrm{Im}$ portantly, we were unable to evaluate the efficiency 
with which people managed their time. For example, one patient may take 10 minutes to do what another could accomplish just as well or better in 5 minutes. In fact, our results suggest some inefficiency; for example, of the women engaging in foot care, one quarter spent 20 minutes or more. Foot care should take most people less than 5 minutes. Our study was also unable to assess the quality of self-care activities, and its cross-sectional design limits its ability to shed light on whether increased time spent results in better health outcomes. A longitudinal study that included health outcomes may better address these important issues.

Notably, this sample of managed care patients may not be typical of all diabetes patients. For example, we included no Medicaid recipients or uninsured people, and we sampled only from one region in the United States. In addition, some potentially clinically important findings were of borderline statistical significance, and larger samples could clarify these findings. Our results should be confirmed in other settings.

In conclusion, we report that these diabetes patients spent considerable time engaged in self-care activities. Despite willingness by 3 quarters to spend at least 20 minutes daily, one third to one half were omitting important individual elements of self-care. In many cases, the mix of activities patients were choosing to perform during self-care time may not have been optimal to maximize health. Patients who self-tested were more likely to engage in other self-care activities, and if they did so, they were more likely to spend more time doing them. These findings have important implications for collaborative clinical practice and care coordination efforts.

\section{Appendix}

\section{Survey Questions about Patient Time}

Think about the extra time you yourself spend taking care of your diabetes-related health problems (related problems might include high blood pressure, high cholesterol, or heart, and circulation problems). If you spend no extra time, please indicate "0" minutes. On a typical day, how many extra minutes do you spend:
A. Caring for your feet?
B. Exercising?

minutes minutes
C. Shopping for and cooking special foods? minutes

We thank the Translating Research Into Action for Diabetes study group and Catarina Kiefe, $\mathrm{PhD}, \mathrm{MD}$ for insightful suggestions.

\section{References}

1. Armstrong N. Coping with diabetes mellitus. A fulltime job. Nurs Clin North Am 1987;22:559-68.

2. Singleton, JK. Caring for themselves: facilitators and barriers to women home care workers who are chronically ill following their care plan. Health Care Women Int 2002;23:692-702.

3. Gold MR, Siegel JE, Russell LB, Milton WC. Costeffectiveness in health and medicine. New York: Oxford University Press; 1996. p. 38-45.

4. Hebert PL, Geiss LS, Tierney EF, Engelgau MM, Yawn BP, McBean AM. Identifying persons with diabetes using Medicare claims data. Am J Med Qual. 1999;14:270-7.

5. Pew Research Center for the people and the press. Survey Reports. [homepage on the Internet]. Polls face growing resistance, but still representative survey experiment shows [released 2004 April 20; cited 2004 November 1]. Available from http://peoplepress.org/reports/display.php3?ReportID $=211$.

6. Frankel LR. The report of the CASRO Task Force on response rates. In: Wiseman F, editor. Improving data quality in a sample survey. Cambridge (MA): Marketing Science Institute; 1983.

7. The American Association for Public Opinion Research. Standard definitions: final dispositions of case codes and outcome rates for surveys. Lenexa (KS): The American Association for Public Opinion Research; 2000.

8. White CC, Powell KE, Hogelin GC. The behavioral risk factor surveys: IV. The descriptive epidemiology of exercise. Am J Prev Med. 1987;3:304-10;

9. Mokdad AH, Bowman BA, Ford ES, et al. The continuing epidemics of obesity and diabetes in the United States. JAMA 2001;286:1195-200.

10. Ware JE, Kosinski M, Keller SD. SF-12: how to score the SF-12 Physical and Mental Health Summary scales. Boston (MA): The Health Institute; 1995.

11. Michigan Diabetes Research and Training Center [homepage on the Internet]. Survey Instruments [updated 2005 April 11; cited 2003 September 23]. Available form: http://www.med.umich.edu/mdrtc/ survey/request.htm\#mnsi.

12. American Diabetes Association: Clinical Practice Recommendations 2003. Diabetes Care 2003;26:S1156.

13. Litzelman DK, Slemenda CW, Langefeld CD, et al. Reduction of lower extremity clinical abnormalities in patients with non-insulin-dependent diabetes 
mellitus. A randomized, controlled trial. Ann Intern Med. 1993;119:36-41.

14. Donabedian A. A founder of quality assessment encounters a troubled system firsthand. Interview by Fitzhugh Mullan. Health Aff (Millwood) 2001;20: 137-41.

15. CDC Diabetes Cost-Effectiveness Group. Cost-effectiveness of intensive glycemic control, intensified hypertension control, and serum cholesterol level reduction for type 2 diabetes. JAMA 2002;287:254251.

16. Johnston M, Rajan M, Mitchinson A, et al. Predictors of self foot care behaviors in veterans at risk for amputation. Diabetes 2003;52:A274.

17. Martin JC, Panicucci CL. Health-related practices and priorities: the health behaviors and beliefs of community-living Black older women. J Gerontol Nurs 1996;22:41-8.

18. US Department of Labor, Bureau of Labor Standards [homepage on the Internet]. Washington DC: US Department of Labor; 2005 [updated 2005 Apr 14; cited 2003 Oct 2]. American Time Use Survey home page; [about 2 screens]. Available from: http:// www.bls.gov/tus/.

19. Juster TF, Stafford FP. The allocation of time: empirical findings, behavioral models, and problems of measurement. J Econ Lit 1991;29:471-522.

20. Kim C, Beckles GL. Cardiovascular disease risk reduction in the Behavioral Risk Factor Surveillance System. Am J Prev Med 2004;27:1-7. 